\title{
GÊNESE DO SERVICCO DE ENFERMAGEM DO HOSPITAL FEDERAL DE BONSUCESSO: CONTRIBUIÇÃO DA ESCOLA ANA NÉRI
}

\author{
Genesis of Nursing Service in Hospital Federal Bonsucesso: contribution of Ana Neri \\ School

\section{Génesis del Servicio de Enfermería en el Hospital Federal de Bonsucesso: contribución de la Escuela Ana Neri}

Camila Pureza Guimarães Silva

Jane Márcia Progianti ${ }^{4}$
Suely de Souza Baptista²

Maria Angélica de Almeida Peres 5 leda de Alencar Barreira ${ }^{3}$

Tânia Cristina Franco Santos ${ }^{6}$

\section{RESUMO}

0 objeto deste estudo é a implantação do Serviço de Enfermagem no Hospital do Instituto de Aposentadoria e Pensões dos Empregados em Transportes e Cargas, atual Hospital Federal de Bonsucesso, no período 1946-1948. Seu objetivo é discutir a participação da Escola Ana Néri nesse processo. Fontes primárias: documentos escritos e depoimentos orais. Fontes secundárias: bibliografias acerca da temática. A abordagem teórico-metodológica apoiou-se em conceitos dos sociólogos Pierre Bourdieu e Maria Cecília Minayo. Evidenciou-se que a contribuição da Escola Ana Néri além de ter sido decisiva para o sucesso da implantação do Serviço de Enfermagem no Hospital do Instituto de Aposentadoria e Pensões dos Empregados em Transportes e Cargas também contribuiu para reforçar sua posição no espaço hospitalar da capital federal no governo Dutra.

Palavras-chave: Enfermagem. História da Enfermagem. Serviço hospitalar de enfermagem. Previdência Social.

\begin{abstract}
The object of this study is the implementation of the Nursing Service at the Hospital of the Institute of Retirement and Pension Employee Transport and Cargo current Federal Bonsucesso Hospital in the period 1946-1948. Your goal is to discuss the participation of Ana Neri School in the process. Primary sources: written documents and oral testimony. Secondary sources: bibliographies on the subject. The theoretical-methodological approach was based on the concepts of the sociologist Pierre Bourdieu and Maria Cecilia Minayo. It was evident that the contribution of Ana Neri School as well as having been crucial to the successful implementation of the Nursing Service at the Hospital of the Institute of Retirement and Pension Employee Transport and Cargo also helped to strengthen its position in the hospital in the federal capital Dutra.
\end{abstract}

Keywords: Nursing. History of Nursing, Nursing Service, Hospital. Social Security.

\section{Resumen}

El objeto de este estudio es la implementación del Servicio de Enfermería en el Hospital del Instituto de Aposentadoria e Pensões dos Empregados em Transportes e Cargas, actual Hospital Federal de Bonsucesso, en el periodo 1946-1948. Su objetivo es discutir la participación de la Escuela Ana Neri en ese proceso. Fuentes primarias: documentos escritos y testimonios orales. Las fuentes secundarias: bibliografías sobre el tema. Los enfoques teóricos y metodológicos se basaron en los conceptos de los sociólogos Pierre Bourdieu y María Cecilia Minayo. Era evidente que la contribución de la Escuela Ana Neri, además de decisiva para la exitosa implementación del Servicio de Enfermería en el Hospital del Instituto de Aposentadoria e Pensões dos Empregados em Transportes e Cargas también contribuyó para fortalecer su posición en el espacio hospitalario de la Capital Federal en el Gobierno Dutra.

Palabras clave: Enfermería. Historia de la Enfermería. Servicio de Enfermería en Hospital. Seguridad Social.

\footnotetext{
${ }^{1}$ Enfermeira do Ministério da Saúde/Hospital Federal de Bonsucesso. Mestranda do Programa de Pós-Graduação da Escola de Enfermagem Anna Nery (EEAN)/Universidade Federal do Rio de Janeiro (UFRJ). Membro do Núcleo de Pesquisa de História da Enfermagem Brasileira (Nuphebras). NilópolisRJ. Brasil. E-mail: milacami@ig.com.br; ${ }^{2}$ Doutora em História da Enfermagem. Docente do Programa de Pós-Graduação da EEAN/UFRJ. Membro fundador do Nuphebras. Rio de Janeiro- RJ. Brasil. E-mail: suelybaptista@openlink.com.br; ${ }^{3}$ Doutora em História da Enfermagem. Docente do Programa de PósGraduação da EEAN/UFRJ. Membro fundador do Nuphebras. Pesquisadora CNPq. Rio de Janeiro-RJ. Brasil. E-mail: iedabarreira@openlink.com.br; ${ }^{4}$ Doutora em História da Enfermagem. Professora Adjunta da Faculdade de Enfermagem da Universidade do Estado do Rio de Janeiro. Vice-Líder do Núcleo de Estudos e Pesquisas de Enfermagem, Mulher, Saúde e Sociedade. Rio de Janeiro- RJ. Brasil. E-mail: jmprogi@uol.com.br; ${ }^{5}$ Doutora em História da Enfermagem. Professora Adjunta do Departamento de Enfermagem Fundamental da EEAN/UFRJ. Membro do Nuphebras. Rio de Janeiro- RJ. Brasil. E-mail:aguaonda@uol.com.br; ${ }^{6}$ Pós-Doutora em História da Enfermagem. Professora Associada do Departamento de Enfermagem Fundamental da EEAN/UFRJ. Membro Fundador do Nuphebras. Pesquisadora CNPq. Rio de Janeiro- RJ. Brasil. E-mail: taniacristinafsc@terra.com.br
} 


\section{INTRODUÇÃO}

Este estudo tem como objeto a implantação do Serviço de Enfermagem no Hospital do Instituto de Aposentadoria e Pensões dos Empregados em Transportes e Cargas (IAPETC) - atual Hospital Federal de Bonsucesso). 0 contexto sóciohistórico no qual se insere o estudo é o da segunda metade da década de 1940, no Rio de Janeiro, à época capital federal, na presidência do general Eurico Gaspar Dutra (1946-1950).

Com o fim da ditadura do Estado Novo de Vargas (1937-1945), os trabalhadores incrementaram a luta contra a rígida restrição de despesas impostas às instituições e por melhorias previdenciárias no modelo assistencial de saúde. Diante da nova conjuntura política, o governo e os dirigentes dos Institutos de Aposentadoria e Pensões se viram pressionados a atender algumas das reivindicações dos trabalhadores, visando minimizar as tensões sociais. ${ }^{1}$

Foi assim que, nas décadas de 1940 e 1950, a política de saúde visou atender prioritariamente as necessidades dos trabalhadores envolvidos com o processo de industrialização, o que favoreceu, no Brasil, a criação dos chamados hospitais modernos. Esses hospitais eram tecnicamente mais sofisticados e, por consequência, mais exigentes quanto à qualificação e diversidade dos profissionais de saúde que neles iriam trabalhar. ${ }^{2}$

A criação de hospitais deste tipo passou a exigir um maior quantitativo de pessoal de enfermagem, o que demandou a criação de escolas para enfermeiras nas diversas regiões do país e a inserção de um novo agente no campo, o auxiliar de enfermagem. Nesse sentido, em 1941, na gestão da diretora Laís Netto dos Reys, a Escola Ana Néri (EAN) iniciou o seu primeiro curso para formação de auxiliares de enfermagem. Esse curso, com duração de 18 meses, visava atender mais rapidamente as necessidades hospitalares no que se refere ao pessoal de enfermagem, uma vez que a duração do curso de formação de enfermeiras era de 36 meses. $^{3}$

Em 1947, diante da situação de crise da saúde no país, o Ministro do Trabalho Indústria e Comércio, Dr. Morvan Dias de Figueiredo, cumprindo ordens do presidente da República, estabeleceu um plano político, em conjunto com o presidente do IAPETC, Dr. Hilton Santos. Esse plano compreendia a construção de um grande hospital na capital federal e outros, de menor porte, nos seguintes estados: Bahia, Pernambuco, São Paulo e Rio Grande do Sul; criando, desta forma, uma rede hospitalar para os contribuintes do IAPETC. 0 Hospital do IAPETC, sediado na capital da República, uma instituição de grande porte para os padrões da época, foi inaugurado no dia 31 de janeiro de 1948., ${ }^{1,4}$

Para que o hospital moderno pudesse funcionar, era essencial a contribuição de um grupo de enfermeiras diplomadas nos moldes da EAN, e em número suficiente para garantirem um alto padrão de qualidade assistencial. Como a
EAN era o padrão oficial para o ensino da enfermagem no Brasil, detinha posição de destaque no campo da educação. 0 poder a ela outorgado pelo Estado, mediante o Decreto $n^{0}$ 20.109/1931, Ihe permitia indicar enfermeiras para inspecionar e controlar tudo o que se referia à enfermagem brasileira, garantindo-lhe o poder de enunciar o discurso autorizado acerca do ensino de enfermagem no país. . $^{5-6}$

Por isso mesmo seria de se esperar que os dirigentes dos hospitais modernos procurassem o apoio da EAN para a organização e o funcionamento daqueles hospitais. Tanto que, das primeiras vinte enfermeiras que integraram o quadro de funcionários do Hospital do IAPETC, dezoito eram diplomadas pela EAN e duas pela Escola de Enfermeiras Luiza de Marillac; a enfermeira responsável pela organização e implantação do Serviço de Enfermagem desse hospital foi a Prof ${ }^{\mathrm{a}}$ da EAN Cecilia Mouniere Pecego, diplomada pela EAN em 1945 e cedida por esta Escola, em 1947, para tal empreendimento. Também os primeiros vinte e cinco auxiliares de enfermagem que iriam trabalhar no Hospital do IAPETC foram formados pela EAN. ${ }^{7-9}$

Diante do exposto, o objetivo desse estudo é discutir a participação da Escola Ana Néri na organização e implantação do Serviço de Enfermagem do Hospital do IAPETC.

Este estudo contribui tanto para a construção de uma parcela da história da enfermagem como para a história das mulheres na sociedade brasileira, mediante a coleta e organização de documentos referentes ao recorte temporal, os quais estão disponibilizados na Biblioteca do atual Hospital Federal de Bonsucesso, no Centro de Documentação da EEAN/ UFRJ (CDOC/EEAN) e no Centro de Memória Matilde Nina da Faculdade de Enfermagem Luiza de Marillac/São Camilo para que futuros pesquisadores realizem novas pesquisas acerca da temática para o melhor desenvolvimento e compreensão da nossa profissão.

\section{MÉTODO}

Estudo histórico-social vinculado à dissertação de mestrado intitulada Serviço de Enfermagem do Hospital do IAPETC: de laico a religioso- 1946-1951, a qual vem sendo desenvolvida junto ao Programa de Pós-Graduação da EEAN/ UFRJ.

0 recorte temporal engloba o período 1946-1948. 0 marco inicial refere-se ao primeiro contato do diretor do Hospital do IAPETC com a diretora da EAN para tratar da organização do Serviço de Enfermagem naquele hospital, e o marco final corresponde ao primeiro ano de implantação do Serviço de Enfermagem no referido hospital.

As fontes primárias incluem: reportagens sobre 0 dia da inauguração do hospital publicadas em jornais da época, consultadas nos arquivos da Biblioteca Nacional; dossiês das primeiras enfermeiras que trabalharam no Hospital do IAPETC; dois depoimentos orais de Cecília Pecego Coelho: um pertencente ao Acervo de História Oral 
do CDOC/EEAN e outro coletado pela primeira autora deste artigo, constantes do acervo do CDOC/EEAN.

Os procedimentos para a coleta de dados foram análise documental e entrevistas. Dentro do universo da história oral, foi utilizada a modalidade da história oral temática, que permite o registro de testemunhos e 0 acesso às "histórias dentro da história" e, dessa forma amplia as possibilidades de interpretação do passado. ${ }^{10}$

As entrevistas foram gravadas em gravador digital (MP3) e posteriormente transcritas, sendo o texto encaminhado ao entrevistado para que ele realizasse 0 ato de conferência e validação, garantindo a doação e o uso posterior do documento através de assinatura do Termo de Cessão ao CEDOC/EEAN. Os depoentes foram previamente informados acerca do objeto de estudo e consultados em relação ao interesse de participar. Todos receberam uma cópia do Termo de Consentimento Livre e Esclarecido, conforme a Resolução 196/96, com detalhes sobre o tema, que foi assinado e devolvido à pesquisadora.

Os instrumentos de coleta de dados foram: roteiro de entrevista semiestruturada, adaptado à peculiaridade de cada agente do estudo e ficha documental que contém a identificação, análise técnica, análise do conteúdo e transcrição e síntese interpretativa do documento, a qual possibilitou relacionar o texto ao contexto histórico. ${ }^{10}$

As fontes secundárias incluíram bibliografias pertinentes à temática em estudo, como livros de história da enfermagem, história do Brasil, teses de doutorado, dissertações de mestrado e artigos publicados.

Os conceitos da Teoria do Mundo Social do sociólogo francês Pierre Bourdieu, tais como: habitus, capital social, cultural, científico e simbólico, campo e espaço social e poder simbólico foram utilizados na leitura e análise do corpus documental. Seu argumento central é o de que as práticas sociais são estruturadas, isto é, apresentam propriedades típicas da posição social de quem as produz. Para este autor, a posição ocupada no espaço social, ou seja, na estrutura de distribuição de diferentes tipos de capital, que muitas vezes são utilizados como armas simbólicas, orienta as representações desse espaço e as tomadas de posição nas lutas com a finalidade de manutenção ou modificação dele. Sendo assim, as marcas das posições sociais, os códigos que expressam tais marcas, bem como as estratégias de reprodução dessas marcas sociais permitiram a compreensão do Hospital do IAPETC, recorte espacial deste estudo, como uma estrutura social e objetiva, onde o habitus profissional das enfermeiras da EAN, entendido como um sistema de disposições incorporadas, operou como um sistema estruturante, ou seja, como princípio gerador e estruturador das práticas e das representações, conferindo à EAN a possibilidade de enunciar o discurso autorizado acerca da estruturação do serviço de enfermagem nesse Hospital. ${ }^{6}$
Dessa forma, os procedimentos de análise e interpretação, como classificação e contextualização dos dados, possibilitaram a discussão dos resultados à luz dos conceitos teóricos de apoio. 0 projeto que deu origem a este estudo foi aprovado pelo Comitê de Ética e Pesquisa do Hospital Federal de Bonsucesso em 12 de abril de 2010 (Protocolo:CEP-HGB 08/10).

\section{RESULTADO E DISCUSSÃO - CONTRIBUIÇÃO DA EAN PARA A ORGANIZAÇÃO E IMPLANTAÇÃO DO SERVIÇO DE ENFERMAGEM NO HOSPITAL DO IAPETC}

Em meados de 1946, o IAPETC encarregou o médico Osvaldo Correa de Araúijo, indicado pelo presidente do Instituto para dirigir o futuro Hospital do IAPETC, para negociar com Laís Netto dos Reys, diretora da EAN, a composição do corpo de enfermagem do Hospital. Esse encontro foi intermediado por Cecilia Pecego, que era sobrinha neta do Dr. Osvaldo e professora da EAN. ${ }^{7-9}$

A partir daí foi estabelecida uma forte aliança entre a EAN e o IAPETC. A primeira estratégia dessas instituições visando ao futuro funcionamento do Hospital do IAPETC foi a organização de um curso para a formação de vinte e cinco auxiliares de enfermagem, os quais deveriam integrar a primeira equipe do referido hospital. Evidencia-se, portanto, que no interior dos campos, diferentes alianças mais ou menos duradouras podem ser instauradas, como, por exemplo, na educação e na saúde. ${ }^{6-9}$

Para o direcionamento pedagógico do curso, a EAN utilizou-se de agentes que já tinham acumulado capital em lutas passadas, como é o caso das professoras Rosaly Taborda, diplomada pela EAN em 1931, a qual, entre outras coisas, organizou o Serviço de Enfermagem do Hospital Servidores do Estado e foi diretora da Escola de Enfermagem de Manaus (1949-1954) e Elvira De Felice, diplomada pela EAN em 1945 e que neste mesmo ano ingressou nesta escola na qualidade de professora, alcançando ao longo de sua carreira acadêmica a posição de professora titular. ${ }^{7}$ Nesse sentido, os agentes sociais incorporam um habitus gerador (disposições adquiridas pela experiência) que variam no tempo e no espaço. Do berço ao túmulo absorvemos (reestruturamos) nossos habitus, condicionando as aquisições mais novas pelas mais antigas. ${ }^{6,12}$

Vale destacar que apesar de o Curso de Auxiliar de Enfermagem ter sido criado na EAN em 1941, sua regulamentação, tão defendida por Laís Netto dos Reys, só ocorreu com a promulgação da Lei n ${ }^{0} 775 / 1949$. Esta mesma Lei retirou da EAN a prerrogativa de Escola Oficial Padrão., 3

Em agosto de 1947, cinco meses antes da inauguração do Hospital do IAPETC, a pedido do dr. Osvaldo, Cecília Pecego, foi cedida pela diretora da EAN para organizar o Serviço de Enfermagem desse Hospital: "Assim que me formei fui ser instrutora do Curso de Auxiliar de Enfermagem. Em 1946 eu já 
estava como professora da EAN e em 1947 iniciei minhas atividades no IAPETC". 8

Era notório que Cecília Pecego acumulava capitais importantes, entre eles, capital cultural institucionalizado, pois era diplomada pela Escola Oficial Padrão, além de exercer atividades docentes na EAN. Desta forma, continuava atualizando o habitus profissional adquirido durante sua formação como enfermeira. Cecília Pecego também era portadora de um importante capital social; era proveniente de família ilustre (constituída de políticos e médicos renomados) do Distrito Federal, tinha grau de parentesco com o futuro diretor do Hospital do IAPETC e um ótimo relacionamento com Laís Netto dos Reys, ocupando, portanto uma posição estratégica no campo em estudo.

A afinidade entre Cecília Pecego e Laís Netto dos Reys advinha tanto do habitus profissional, pois eram diplomadas pela Escola Oficial Padrão e exerciam atividades docentes na EAN, como do habitus religioso, uma vez que ambas, por serem católicas convictas tinham forte aliança com a Igreja Católica. Tanto que Cecília Pecego integrou a primeira diretoria da União Católica de Enfermeiras do Brasil (UCEB- criada em 4 de abril de 1948, com o objetivo de estabelecer maior aproximação e entendimento entre enfermeiras católicas laicas e enfermeiras religiosas) na qualidade de vice-presidente, e Laís Netto dos Reys foi aclamada presidente de honra desta instituição. ${ }^{11}$

Logo que assumiu suas atividades no IAPETC, Cecília Pecego iniciou um curso de treinamento para os cinco auxiliares e atendentes de enfermagem que trabalhavam no ambulatório deste instituto, pois eles seriam transferidos do ambulatório para o hospital, assim fosse inaugurado. 0 curso teve duração de um mês e meio e consistia no treinamento de procedimentos técnicos de enfermagem, como aplicação de injeções, realização de curativos, ou seja, assistência de enfermagem, desde que 0 paciente chegava até o encaminhamento à consulta médica-9 ${ }^{8-9}$.

No período de organização do Hospital, agosto de 1947 a janeiro de 1948, Cecília Pecego comparecia diariamente ao prédio sede do IAPETC, onde funcionava também o ambulatório, para tomar diversas providências, dentre elas participar da seleção e compra de equipamentos, inclusive os provenientes do exterior, e verificar a confecção dos uniformes dos funcionários de enfermagem, como a escolha do tecido que seria comprado e o modelo de uniforme para enfermeiras e auxiliares. ${ }^{8-9}$

Cabe ressaltar a preocupação de Cecília Pecego na distinção dos agentes de enfermagem no campo pela utilização de modelos de uniforme diferentes. 0 espaço social para Bourdieu tende a se retraduzir no espaço físico, sob a forma de um arranjo de agentes e propriedades, onde as divisões e distinções do social em categorias de alto e baixo exprime-se real e simbolicamente, no espaço físico apropriado como espaço social reificado. ${ }^{6}$

A partir do final de novembro de 1947, Cecília Pecego dividia seu tempo entre o prédio sede do IAPETC, situado na Av. Venezuela - Centro da Cidade do Rio de Janeiro e o do
Hospital do IAPETC, situado na Av. Londres, Bairro de Bonsucesso, onde já estavam sendo disponibilizados materiais, como camas, macas, mesas, equipamentos. Nesta época, devido ao grande volume de trabalho e à proximidade da inauguração do Hospital, Cecília Pecego indicou e o IAPETC contratou as enfermeiras: Nilza Moraes Passos, Maria Eleusis Riomar e Fernanda Walter Passos, todas diplomadas pela EAN, sendo as duas primeiras da classe de 1945 e a última da classe de 1946..$^{7-9}$

A respeito da solenidade de inauguração do referido hospital, estiveram presentes as mais altas autoridades, inclusive o Presidente da República Eurico Gaspar Dutra, Ministros e Secretários de Estado, como Dr. Morvan Figueiredo e Clovis Pestana, respectivamente Ministro do Trabalho, Indústria e Comércio e da Aviação, o Presidente do IAPETC, Hilton Santos, e o Presidente do Sindicato do Empregados em Transportes e Cargas, Melquíades de Oliveira Bastos. Segundo Cecília Pecego, a inauguração do hospital foi amplamente apoiada pelos contribuintes do IAPETC: "no dia da inauguração todos estiveram presentes acompanhados de suas famílias, demonstrando grande satisfação". Na oportunidade foi celebrada uma missa de Ação de Graças por D. Jorge Marcos de Oliveira, bispo auxiliar do Rio de Janeiro. ${ }^{4-5,9}$

A presença de figuras prestigiosas da sociedade brasileira, inclusive da autoridade máxima do país na inauguração do hospital emprestou à cerimônia o efeito de 'magia social', uma vez que a eficácia simbólica da nomeação de uma pessoa ou instituição é proporcional à autoridade daquele que a enuncia, mediante 0 reconhecimento de seu poder. ${ }^{12}$

A igreja desenvolve estratégias visando participar da ordem política e da sua manutenção, pelo inculcamento dos esquemas de percepção, pensamentos e ações conferidos às estruturas políticas, conferindo a legitimação suprema, capaz de instaurar e restaurar o consenso que permeia a ordem do mundo e impõe e inculca os pensamentos comuns. A cerimônia religiosa constitui uma ação simbólica que utiliza a eficácia simbólica dos símbolos religiosos, reforçando, portanto, a crença efetiva em sua eficácia. Portanto, parece que a presença da Igreja na inauguração do Hospital do IAPETC teve como objetivo o for talecimento e estabelecimento de importantes alianças visando manter ou ampliar seu poder. ${ }^{6}$

Apesar de as quatro enfermeiras e dos vinte e cinco auxiliares de enfermagem conseguirem organizar o hospital para o dia da inauguração, este começou a funcionar sem as condições necessárias:

Ainda no dia da inauguração, quando foi mais tarde, o diretor me chamou e disse que o Presidente da República queria ver um doente na cama. Dali a pouco chegou um senhor na ambulância (...) o doente era diabético e não havia insulina e nem seringa de insulina; mandamos comprar 
imediatamente na farmácia em frente. Felizmente era só isso que o doente tinha, porque se fosse mais grave (...). Acho que o oxigênio estava chegando naquele dia. Nas primeiras semanas iam sendo admitidos pacientes com diagnóstico mais simples (Cecilia Pecego).

0 Hospital do IAPETC foi inaugurado em um contexto sócio-político de ênfase na construção de hospitais e ambulatórios por parte dos institutos previdenciários. A área da assistência médica foi objeto de especial atenção do governo, o que veio ao encontro de diversas reivindicações trabalhistas que, por consequência, ao serem atendidas, constituíam, para o governo, estratégias para ganhos político-eleitorais. ${ }^{1}$

Cecília Pecego ainda lembra que a "implantação do Serviço de Enfermagem não foi tão fácil" e comenta que enfrentou uma série de dificuldades: "0 material não foi de tão boa qualidade; o material de enfermagem foi bastante antigo, pois eles aproveitaram materiais do Ambulatório" ${ }^{8-9}$.

Cecilia Pecego também relata outro tipo de dificuldade, qual seja, a de ter que se responsabilizar por outros serviços como o de Farmácia e o de Nutrição, os quais interferiram diretamente na assistência de enfermagem ao paciente até a chegada de profissionais para assumirem tais serviços. ${ }^{9}$

Cecília Pecego aponta mais uma dificuldade, agora com relação à administração financeira dos gastos do Serviço de Enfermagem:

O tesoureiro do hospital queria que as enfermeiras mandassem todos os dias, de cada paciente, toda a medicação que eles tomaram e eu disse: 'Não é possivel. Eu não vou colocar uma enfermeira para escrever o dia inteiro quantas ampolas o doente usou'. Então conversamos e fizemos um caderno grande, onde se passavam todas as ordens médicas, determinando os horários e checávamos ali. Combinei com ele de mandar duas cópias com papel carbono; a original ficava na enfermaria e duas cópias iam para a tesouraria e lá eles faziam a contagem das medicações (Cecilia Pecego).

Contudo, o que parece é que Cecilia Pecego e seu grupo de enfermeiras conseguiram manter-se em uma posição de não aceitar o que era muitas vezes imposto e conseguiram êxito nessas lutas.

Apesar da distância temporal de mais de sessenta anos, Cecília Pecego fez a seguinte reflexão:

Era muito complicado, mas pelo menos salvamos a enfermagem. Foi uma luta muito grande para colocarmos a enfermagem naquilo que era realmente a sua função (...). Não deu para fazer tudo o que eu queria, mas deixei o hospital com a enfermagem organizada, trabalhando nos moldes da ética que nós aprendemos, nos moldes dos serviços de atendimento ao cliente que nós queríamos, defendendo os pontos de vista da enfermagem diante da direção do hospital (Cecilia Pecego).

Sabe-se que no interior de cada um dos espaços sociais estabelecem-se contínuos embates, de diferentes formas, envolvendo os ocupantes das posições dominantes e os ocupantes das posições dominadas. As alianças e as lutas concorrenciais são estabelecidas com o propósito de assegurar e mesmo de ampliar as posições de poder e prestígio no interior dos espaços sociais. ${ }^{6}$ As situações enfrentadas por Cecília Pecego e seu grupo eram questões que envolviam a enfermagem nacional, como, por exemplo, as atribuições da enfermeira, que muito eram discutidas à época. Portanto, isto exigia o estabelecimento de alianças de acordo com os interesses em jogo:

Onosso planejamento foi bem feito, porque nós fizemos um curso de auxiliares e procuramos colocar o máximo possível de enfermeiras da Escola Ana Néri (...). Nós tínhamos um potencial muito grande de antigas alunas, que estavam em outros serviços e queriam ir para o nosso. Foi uma época muito boa para a enfermagem, no sentido de poder colocar enfermeiras diplomadas em todos os setores (Cecília Pecego).

Cabe destacar que naquele momento histórico de implantação do Serviço de Enfermagem no Hospital do IAPETC, Laís Netto dos Reys foi de suma importância, pois era detentora de um extraordinário volume de diversos tipos de capital. E isto em muito deve ter favorecido a articulação de todo aquele processo. Assim, Laís foi ganhando espaço no campo que queria se inserir e inserir a EAN baseando-se na sua percepção individual, na sua forma de pensar e tendo condutas de acordo com essa percepção.

Portanto, evidenciou-se que a contribuição da EAN além de ter sido decisiva para o sucesso da implantação do Serviço de Enfermagem no Hospital do IAPETC também contribuiu para reforçar a posição da EAN no espaço hospitalar da capital federal no governo Dutra.

\section{CONSIDERAÇÕES FINAIS}

A inauguração do Hospital do IAPETC nos moldes preconizados pelo modelo do hospital moderno foi viabilizada por decisivas alianças. 0 hospital era próprio do IAPETC, foi construído com padrões tecnológico avançados para a época e foi uma forma de cumprimento de uma das metas do programa político do governo Dutra (1946-1950).

A aliança política estabelecida entre o primeiro diretor do hospital, o cirurgião geral Osvaldo Correa de Araújo, e o presidente do referido instituto, Dr. Hilton Santos, e com o Governo Dutra, estava diretamente ligada com a aliança com a Igreja Católica, uma vez que, já na inauguração do hospital, podemos constatar a sua presença na figura do Bispo D. Jorge Marcos de Oliveira que realizou uma missa de ação de graças abençoando a solenidade de inauguração, o que se constituiu em uma ação visando lucros simbólicos. 
A inauguração do Hospital do IAPETC também contou com 0 apoio sindical, que mobilizou os empregados em transportes e cargas, tanto que no dia da solenidade houve a presença da população em massa demonstrando forte apoio ao governo e ao presidente do instituto, como também a redução de movimentos populares, que havia aumentado naquele período após o término do Estado Novo (1937-1945), decorrente da insatisfação dos segurados do IAPETC.

Outra aliança de fundamental importância foi a estabelecida entre o IAPETC e a EAN, escola de enfermagem tradicional e criada nos moldes da enfermagem moderna, sendo responsável pelo primeiro corpo de enfermagem do Hospital do IAPETC, qual sejam dezoito enfermeiras diplomadas, vinte e cinco auxiliares de enfermagem e pela cessão da enfermeira para organizar e implantar o Serviço de Enfermagem. A aliança com a EAN foi intermediada pela diretora da Escola à época, Laís Netto dos Reys, portadora de um importante capital social, científico e cultural, a qual tinha forte aliança com a Igreja Católica e com o governo.

Devemos destacar que a enfermeira Cecília Pecego era portadora de diversos tipos de capital que the proporcionaram poder e prestígio no campo da enfermagem, como também uma importante posição no espaço social referente a este estudo.

A primeira constituição do corpo de enfermagem do hospital por profissionais formados pela EAN possibilitou uma organização interna coerente com o conceito de hospital moderno. Certamente, as alianças estabelecidas, a contribuição da EAN e o volume de capital social em jogo foram decisivos para a inauguração do Hospital do IAPETC e para seu pleno funcionamento.

\section{REFERÊNCIAS}

1. Oliveira JAA, Teixeira SMF. (Imp) previdência social: 60 anos de história da Previdência no Brasil. Petrópolis (RJ): Vozes/ Abrasco; 1985.

2. Vaghetti H, Vaz MRC. Década de 40: uma reflexão sobre o contexto sócio-econômico e político brasileiro e sua influência na enfermagem. Texto\&Contexto Enferm. 1998 jan/abr; 7 (I): 9-26.

3. Fonte AS. A Escola de Enfermagem Anna Nery e a nova ordem no campo da educação em enfermagem: 1949 - 1961. [tese]. Rio de Janeiro: Escola de Enfermagem Anna Nery, Universidade Federal do Rio de Janeiro; 2009.

4. Carvalho Junior H. Mais uma obra relevante de assistência social para os trabalhadores. Diário Carioca, Rio de Janeiro, 01 fev.1948. Ano 21, p: $3-4$.

5. BaptistaSS. Trajetória das escolas de enfermagem na sociedade brasileira. Esc Anna Nery. 1997 dez; 1(2): 84-105.
6. Bourdieu P. 0 poder simbólico. $11^{\text {a }}$ ed. Rio de Janeiro: Bertrand Brasil; 2007

7. Coelho CP. A Escola de Enfermagem Anna Nery: sua história, nossas memórias. Rio de Janeiro: Cultura Médica; 1997.

8. Coelho CP. Depoimento de história de vida profissional. Teresópolis, 2006. Entrevista concedida a Renata Lucas Mercês Silva em 25 jan 2006. Conferência e copydesk: leda de Alencar Barreira e Suely de Souza Baptista. [CD-ROM]. Rio de Janeiro: UFRJ/ EEAN/Centro de Documentação; 2006.

9. Coelho CP. Depoimento temático sobre a vida profissional no Hospital do IAPETC. Teresópolis, 2009. Entrevista concedida a Camila Pureza Guimarães da Silva em 30 de maio 2009. [CD-ROM]. Rio de Janeiro: UFR/ EEAN/Centro de Documentação; 2010.

10. Minayo MC. 0 desafio do conhecimento: pesquisa qualitativa em saúde. $7^{\text {a }}$ ed. São Paulo: Hucitec/ABRASCO; 2000

11. Gomes TO, Silva BR, Baptista SS, Almeida Filho AJ. Enfermeiras católicas em busca de melhores posições no campo da educação e da prática em enfermagem nos anos 40 e 50 no Brasil, no século XX. Texto\&Contexto Enferm. 2005 out/dez; 14(4): 506-12.

12. Bourdieu P. A economia das trocas lingüísticas: o que falar quer dizer. $2^{a}$ ed. São Paulo: EDUSP; 1998. 